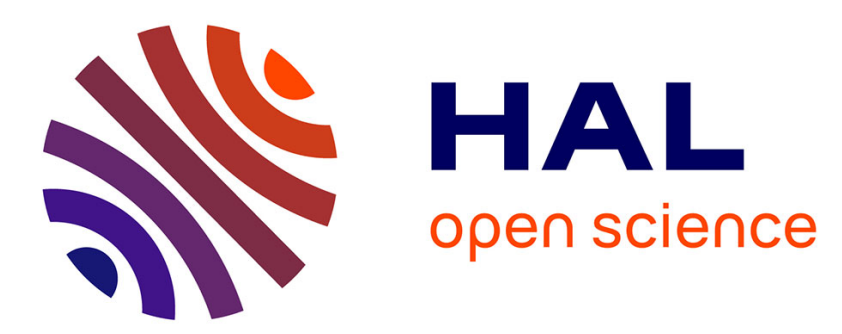

\title{
Sequential Closed-Form Semi-Blind Receiver for Space-Time Coded Multi-Hop Relaying Systems
}

Walter Freitas, Gérard Favier, André L. F. de Almeida

\section{To cite this version:}

Walter Freitas, Gérard Favier, André L. F. de Almeida. Sequential Closed-Form Semi-Blind Receiver for Space-Time Coded Multi-Hop Relaying Systems. IEEE Signal Processing Letters, 2017, 24 (12), pp.1773-1777. hal-01699755

\section{HAL Id: hal-01699755 https://hal.science/hal-01699755}

Submitted on 13 Apr 2018

HAL is a multi-disciplinary open access archive for the deposit and dissemination of scientific research documents, whether they are published or not. The documents may come from teaching and research institutions in France or abroad, or from public or private research centers.
L'archive ouverte pluridisciplinaire HAL, est destinée au dépôt et à la diffusion de documents scientifiques de niveau recherche, publiés ou non, émanant des établissements d'enseignement et de recherche français ou étrangers, des laboratoires publics ou privés. 


\title{
Sequential Closed-Form Semi-Blind Receiver for Space-Time Coded Multi-Hop Relaying Systems
}

\author{
Walter da C. Freitas Jr., Gérard Favier, André L. F. de Almeida, Senior Member, IEEE
}

\begin{abstract}
In this letter, we present a sequential closed-form semi-blind receiver for a one-way multi-hop amplify-and-forward (AF) relaying system. Assuming Khatri-Rao space-time (KRST) coding at each relay, it is shown that the system with $K$ relays can be modeled by means of a generalized nested PARAFAC model. Decomposing this model into $K+1$ third-order PARAFAC models, we develop a closed-form semi-blind receiver for jointly estimating the information symbols and the individual channels, at the destination node. Each step consists of a Khatri-Rao factorization. Parameter identifiability conditions are given, and simulation results are provided to illustrate the effectiveness of the proposed semi-blind receiver.
\end{abstract}

Index Terms-Multi-hop relaying, sequential closed-form semi-blind receiver, space time (ST) coding, amplify-and-forward, generalized nested PARAFAC model.

\section{INTRODUCTION}

Relay nodes will play an important role in 5G communication systems. In such systems, the high-capacity wireless backhaul offers operators an alternative solution to conventional backhauling using multi-hop short-distance communications [1]-[4].

Tensor decompositions have been widely exploited for point-to-point wireless communication systems. The practical motivation for tensor modeling comes from the fact that one can simultaneously benefit from multiple (more than two) signal diversities, like space, time and frequency diversities, for instance. In the context of cooperative wireless communications, few results have been published on tensor-based receivers, and most of them are limited to a single relay scenario. In previous works [5]-[8], the problem of channel estimation for multiple input multiple output (MIMO) relaying systems was considered under a supervised approach. The works [5]-[7] considered one-way two-hop systems, while [8] addressed two-way MIMO relaying systems. In [9] and [10], three-hop relaying systems were studied. Recent works proposed semi-blind receivers for joint symbol and channel estimation. For instance, one can cite the PARAFAC-PARATUCK [11] and the nested PARAFAC receivers [12], [13]. However, the generalization of tensor-based semi-blind receivers to the multi-hop relaying scenario has not been addressed so far to the best of authors' knowledge.

Copyright (c) 2017 IEEE. Personal use of this material is permitted. However, permission to use this material for any other purposes must be obtained from the IEEE by sending a request to pubs-permissions@ieee.org.

Walter da C. Freitas Jr. and André L. F. de Almeida are with the Wireless Telecom Research Group, Department of Teleinformatics Engineering, Federal University of Ceará, Fortaleza, Brazil. (e-mails: \{walter,andre\}@gtel.ufc.br). Gerard Favier is with the I3S Laboratory, University of Nice-Sophia Antipolis (UNS), CNRS, France. (e-mail: favier@i3s.unice.fr). This work was supported by $\mathrm{CNPq}$ and the CAPES/COFECUB project number Ma 830-15.
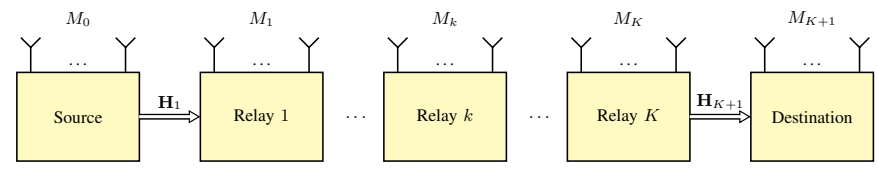

Fig. 1. System model with $K$ relays.

In this paper, we consider a one-way multi-hop relaying MIMO wireless system composed of $K$ relays. Using an AF protocol, each relay retransmits the received signals without decoding. Assuming that the signals are encoded with a simplified Khatri-Rao space-time (KRST) coding scheme [14], it is shown that the signals received at destination satisfy a generalized nested PARAFAC model. By decomposing this model into $K+1$ third-order PARAFAC models, and assuming that the coding matrices are known at the destination node, a closed-form semi-blind receiver based on rank-one matrix approximations is derived for jointly estimating the information symbols and the individual channels.

Notation: Scalars, column vectors, matrices and tensors are denoted by lower-case, boldface lower-case, boldface upper-case, and calligraphic letters, e.g., $\quad a, \mathbf{a}, \mathbf{A}, \mathcal{A}$, respectively. The operator $\operatorname{diag}(\cdot)$ forms a diagonal matrix from its vector argument. The Khatri-Rao and Hadamard products are denoted by $\diamond$ and $*$, respectively. We use the superscripts $T,{ }^{*},{ }^{H},-1, \dagger$ for matrix transposition, complex conjugation, Hermitian transposition, inversion, and Moore-Penrose pseudo inversion, respectively.

Given a third-order tensor $\mathcal{X} \in \mathbb{C}^{I \times J \times K}$, with entries $x_{i, j, k}$, the matrices $\mathbf{X}_{J K \times I}, \mathbf{X}_{K I \times J}$, and $\mathbf{X}_{I J \times K}$ denote tall mode-1, mode- 2 and mode- 3 unfoldings, with $x_{i, j, k}=\left[\mathbf{X}_{I J \times K}\right]_{(i-1) J+j, k}=\left[\mathbf{X}_{J K \times I}\right]_{(j-1) K+k, i}=$ $\left[\mathbf{X}_{K I \times J}\right]_{(k-1) I+i, j}$. A PARAFAC decomposition of $\mathcal{X} \in \mathbb{C}^{I \times J \times K}$, with rank- $R$ and matrix factors $(\mathbf{A}, \mathbf{B}, \mathbf{C})$, will be abbreviated $\|\mathbf{A}, \mathbf{B}, \mathbf{C} ; R\|$. Tall and flat mode-1 matrix unfoldings of $\mathcal{X}$ are respectively given by

$$
\mathbf{X}_{J K \times I}=(\mathbf{B} \diamond \mathbf{C}) \mathbf{A}^{T}=\left(\mathbf{X}_{I \times J K}\right)^{T} \text {. }
$$

Mode-2 and mode-3 unfoldings are easily deduced by permuting $(I, J, K)$ and $(\mathbf{A}, \mathbf{B}, \mathbf{C})$ accordingly.

\section{SySTEM MODEL}

We consider a one-way multi-hop MIMO relaying system, composed of $K$ relays, as illustrated in Figure 1, where $M_{k}$ denotes the number of antennas at node $k$, with $k=$ $0, \cdots, K+1$. The nodes indexed by $k=0$ and $k=K+1$ represent the source and the destination, respectively. The matrix $\mathbf{H}_{k+1} \in \mathbb{C}^{M_{k+1} \times M_{k}}, k=0, \cdots, K$, denotes the channel between nodes $k$ and $k+1$, which is assumed as flat-fading and quasi-static during the total transmission time. 
Let us define the symbol matrix $\mathbf{S} \in \mathbb{C}^{N \times M_{0}}$ containing $N$ data-streams composed of $M_{0}$ symbols that are multiplexed by $M_{0}$ transmit antennas at the source. Source and relays encode the signals to be transmitted with a KRST coding matrix $\mathbf{G}_{k} \in \mathbb{C}^{P_{k} \times M_{k}}$, chosen as a truncated discrete Fourier transform (DFT) matrix [12] implying $\mathbf{G}_{k}^{T} \mathbf{G}_{k}^{*}=\mathbf{I}_{M_{k}}$ for $k=0, \cdots, K$. The processing at the relay nodes follows the AF protocol. Let $\tilde{\mathcal{X}}^{(k)}=\mathcal{X}^{(k)}+\mathcal{N}^{(k)}$ be the noisy received signal tensor at node $k$, the entries of the noise tensor $\mathcal{N}^{(k)}$ being zero-mean circularly-symmetric complex-valued Gaussian random variables.

\section{A. Case with two relays}

In this section, we consider the particular case with two relays $(K=2)$. In the next section, we present a generalization to an arbitrary number $K$ of relays. The signals received at relay-1 define a third-order tensor $\tilde{\mathcal{X}}^{(1)} \in \mathbb{C}^{M_{1} \times P_{0} \times N}$ satisfying a PARAFAC model $\left\|\mathbf{H}_{1}, \mathbf{G}_{0}, \mathbf{S} ; M_{0}\right\|$, a flat mode-1 unfolding of which is given by

$$
\tilde{\mathbf{X}}_{M_{1} \times P_{0} N}^{(1)}=\mathbf{H}_{1}\left(\mathbf{G}_{0} \diamond \mathbf{S}\right)^{T}+\mathbf{N}_{M_{1} \times P_{0} N}^{(1)} .
$$

Relay-1 re-encodes the received signals using a KRST code $\mathbf{G}_{1}$. The signals received at relay-2 define a fourth-order tensor $\tilde{\mathcal{X}}^{(2)} \in \mathbb{C}^{M_{2} \times P_{1} \times P_{0} \times N}$ which satisfies the following equations

$$
\begin{aligned}
\tilde{\mathbf{X}}_{M_{2} \times P_{1} P_{0} N}^{(2)} & =\mathbf{H}_{2}\left(\mathbf{G}_{1} \diamond \tilde{\mathbf{X}}_{P_{0} N \times M_{1}}^{(1)}\right)^{T}+\mathbf{N}_{M_{2} \times P_{1} P_{0} N}^{(2)},(3) \\
& =\mathbf{H}_{2}\left(\mathbf{G}_{1} \diamond\left(\mathbf{G}_{0} \diamond \mathbf{S}\right) \mathbf{H}_{1}^{T}\right)^{T} \\
& +\mathbf{H}_{2}\left(\mathbf{G}_{1} \diamond \mathbf{N}_{P_{0} N \times M_{1}}^{(1)}\right)^{T}+\mathbf{N}_{M_{2} \times P_{1} P_{0} N}^{(2)} .
\end{aligned}
$$

The information part in (4) corresponds to a nested PARAFAC model [15], whereas Eq. (3) is a PARAFAC model $\left\|\mathbf{H}_{2}, \mathbf{G}_{1}, \tilde{\mathbf{X}}_{P_{0} N \times M_{1}}^{(1)} ; M_{1}\right\|$ for the contracted form of $\tilde{\mathcal{X}}^{(2)}$ resulting from a combination of its last two modes $\left(p_{0}, n\right)$.

After KRST coding at relay- 2 and transmission through the channel $\mathbf{H}_{3}$, the signals received at the destination define a fifth-order tensor $\tilde{\mathcal{X}}^{(3)} \in \mathbb{C}^{M_{3} \times P_{2} \times P_{1} \times P_{0} \times N}$, which can be written as

$\tilde{\mathbf{X}}_{M_{3} \times P_{2} P_{1} P_{0} N}^{(3)}=\mathbf{H}_{3}\left(\mathbf{G}_{2} \diamond \tilde{\mathbf{X}}_{P_{1} P_{0} N \times M_{2}}^{(2)}\right)^{T}+\mathbf{N}_{M_{3} \times P_{2} P_{1} P_{0} N}^{(3)}$.

Replacing $\tilde{\mathbf{X}}_{P_{1} P_{0} N \times M_{2}}^{(2)}$, and $\tilde{\mathbf{X}}_{P_{0} N \times M_{1}}^{(1)}$ by their expressions (3) and (2), we obtain the following matrix unfolding for $\tilde{\mathcal{X}}^{(3)}$

$$
\begin{array}{r}
\tilde{\mathbf{X}}_{M_{3} \times P_{2} P_{1} P_{0} N}^{(3)}=\mathbf{H}_{3}\left[\mathbf{G}_{2} \diamond\left(\mathbf{G}_{1} \diamond\left(\mathbf{G}_{0} \diamond \mathbf{S}\right) \mathbf{H}_{1}^{T}\right) \mathbf{H}_{2}^{T}\right]^{T} \\
+\underbrace{\mathbf{H}_{3}\left(\mathbf{G}_{2} \diamond\left(\mathbf{G}_{1} \diamond \mathbf{N}_{P_{0} N \times M_{1}}^{(1)}\right) \mathbf{H}_{2}^{T}\right)^{T}}_{\mathbf{N}_{M_{3} \times P_{2} P_{1} P_{0} N}^{(1 \mapsto 3}} \\
+\underbrace{\mathbf{H}_{3}\left(\mathbf{G}_{2} \diamond \mathbf{N}_{P_{1} P_{0} N \times M_{2}}^{(2)}\right)^{T}}_{\mathbf{N}_{M_{3} \times P_{2} P_{1} P_{0} N}^{(2 \leftrightarrow)}}+\mathbf{N}_{M_{3} \times P_{2} P_{1} P_{0} N}^{(3)} .
\end{array}
$$

The information part in (6) corresponds to a (generalized) fifth-order nested PARAFAC model. The terms $\mathbf{N}_{M_{3} \times P_{2}}^{(1 \mapsto 3)}$ and $\mathbf{N}_{M_{3} \times P_{2} P_{1} P_{0} N}^{(2 \mapsto 3)}$ are the noise contributions from relays 1 and 2 received at the destination. Eq. (5) is a PARAFAC model $\left\|\mathbf{H}_{3}, \mathbf{G}_{2}, \tilde{\mathbf{X}}_{P_{1} P_{0} N \times M_{2}}^{(2)} ; M_{2}\right\|$ for the contracted form of $\tilde{\mathcal{X}}^{(3)}$ obtained by combining its last three modes $\left(p_{1}, p_{0}, n\right)$.
From now on, we consider a noiseless formulation due to space limitation. Another unfolding of the PARAFAC model (5) is

$$
\mathbf{X}_{M_{3} P_{2} \times P_{1} P_{0} N}^{(3)}=\left(\mathbf{H}_{3} \diamond \mathbf{G}_{2}\right) \mathbf{X}_{M_{2} \times P_{1} P_{0} N}^{(2)} .
$$

Replacing $\mathbf{X}_{M_{2} \times P_{1} P_{0} N}^{(2)}$ by its expression (3) leads to

$$
\begin{aligned}
\mathbf{X}_{M_{3} P_{2} \times P_{1} P_{0} N}^{(3)} & =\left(\mathbf{H}_{3} \diamond \mathbf{G}_{2}\right) \mathbf{H}_{2}\left(\mathbf{G}_{1} \diamond \mathbf{X}_{P_{0} N \times M_{1}}^{(1)}\right)^{T} \\
& =\mathbf{H}_{M_{3} P_{2} \times M_{1}}^{(1 \mapsto 3)}\left(\mathbf{G}_{1} \diamond \mathbf{X}_{P_{0} N \times M_{1}}^{(1)}\right)^{T}
\end{aligned}
$$

where

$$
\mathbf{H}_{M_{3} P_{2} \times M_{1}}^{(1 \mapsto 3)}=\left(\mathbf{H}_{3} \diamond \mathbf{G}_{2}\right) \mathbf{H}_{2}
$$

is a matrix unfolding of the third-order effective channel tensor $\mathcal{H}^{(1 \mapsto 3)} \in \mathbb{C}^{M_{3} \times P_{2} \times M_{1}}$ linking the relay-1 and the destination (node-3). From (9), we can conclude that the contracted form of $\mathcal{X}^{(3)}$ resulting from the combination of its first two modes $\left(m_{3}, p_{2}\right)$ and its last two modes $\left(p_{0}, n\right)$, satisfies a PARAFAC model $\left\|\mathbf{H}_{M_{3} P_{2} \times M_{1}}^{(1 \mapsto 3)}, \mathbf{G}_{1}, \mathbf{X}_{P_{0} N \times M_{1}}^{(1)} ; M_{1}\right\|$.

Permuting the matrix factors in (9) gives the following unfolding of $\mathcal{X}^{(3)}$

$$
\mathbf{X}_{M_{3} P_{2} P_{1} \times P_{0} N}^{(3)}=\left(\mathbf{H}_{M_{3} P_{2} \times M_{1}}^{(1 \mapsto 3)} \diamond \mathbf{G}_{1}\right) \mathbf{X}_{M_{1} \times P_{0} N}^{(1)} .
$$

By replacing $\mathbf{X}_{M_{1} \times P_{0} N}^{(1)}$ by its expression (2), we get

$$
\begin{aligned}
\mathbf{X}_{M_{3} P_{2} P_{1} \times P_{0} N}^{(3)} & =\left(\mathbf{H}_{M_{3} P_{2} \times M_{1}}^{(1 \mapsto 3)} \diamond \mathbf{G}_{1}\right) \mathbf{H}_{1}\left(\mathbf{G}_{0} \diamond \mathbf{S}\right)^{T}, \\
& =\mathbf{H}_{M_{3} P_{2} P_{1} \times M_{0}}^{(0 \mapsto 3)}\left(\mathbf{G}_{0} \diamond \mathbf{S}\right)^{T},
\end{aligned}
$$

where

$$
\mathbf{H}_{M_{3} P_{2} P_{1} \times M_{0}}^{(0 \mapsto 3)}=\left(\mathbf{H}_{M_{3} P_{2} \times M_{1}}^{(1 \mapsto 3)} \diamond \mathbf{G}_{1}\right) \mathbf{H}_{1}
$$

is an unfolding of the fourth-order effective channel tensor $\mathcal{H}^{(0 \mapsto 3)} \in \mathbb{C}^{M_{3} \times P_{2} \times P_{1} \times M_{0}}$ linking the source and the destination. Eq. (12) corresponds to the PARAFAC model $\left\|\mathbf{H}_{M_{3} P_{2} P_{1} \times M_{0}}^{(0 \mapsto 3)}, \mathbf{G}_{0}, \mathbf{S} ; M_{0}\right\|$ of a contracted form of $\mathcal{X}^{(3)}$ obtained by combining its modes $\left(m_{3}, p_{2}, p_{1}\right)$.

Permuting the matrix factors in (12), (13), and (10) gives the following unfoldings of $\mathcal{X}^{(3)}, \mathcal{H}^{(0 \mapsto 3)}$, and $\mathcal{H}^{(1 \mapsto 3)}$

$$
\begin{aligned}
\mathbf{X}_{N M_{3} P_{2} P_{1} \times P_{0}}^{(3)} & =\left(\mathbf{S} \diamond \mathbf{H}_{M_{3} P_{2} P_{1} \times M_{0}}^{(0 \mapsto 3)}\right) \mathbf{G}_{0}^{T}=\mathbf{R G}_{0}^{T}, \\
\mathbf{H}_{M_{3} P_{2} M_{0} \times P_{1}}^{(0 \mapsto 3)} & =\left(\mathbf{H}_{M_{3} P_{2} \times M_{1}}^{(1 \mapsto 3)} \diamond \mathbf{H}_{1}^{T}\right) \mathbf{G}_{1}^{T}=\mathbf{Q G}_{1}^{T}, \\
\mathbf{H}_{M_{3} M_{1} \times P_{2}}^{(1 \mapsto 3)} & =\left(\mathbf{H}_{3} \diamond \mathbf{H}_{2}^{T}\right) \mathbf{G}_{2}^{T}=\mathbf{Z G}_{2}^{T} .
\end{aligned}
$$

We assume that the coding matrices $\mathbf{G}_{k}$, for $k=0,1,2$, are known at the destination node.

Decomposing the fifth-order nested PARAFAC model (6) into three third-order PARAFAC models represented by Eq. (14)-(16) allows us to derive a three-step closed-form semi-blind receiver to estimate the symbol matrix $\mathbf{S}$ and the channel matrices $\mathbf{H}_{k}, k=1,2,3$. Indeed, using the column orthonormality of the code matrices $\mathbf{G}_{k}, k=0,1,2$, the least squares (LS) estimates of $(\mathbf{R}, \mathbf{Q}, \mathbf{Z})$ can be successively calculated as

$$
\begin{aligned}
\hat{\mathbf{R}} & =\tilde{\mathbf{X}}_{N M_{3} P_{2} P_{1} \times P_{0}}^{(3)} \mathbf{G}_{0}^{*}, \\
\hat{\mathbf{Q}} & =\hat{\mathbf{H}}_{M_{3} P_{2} M_{0} \times P_{1}}^{(0 \mapsto 3)} \mathbf{G}_{1}^{*}, \\
\hat{\mathbf{Z}} & =\hat{\mathbf{H}}_{M_{3} M_{1} \times P_{2}}^{(1 \mapsto 3)} \mathbf{G}_{2}^{*},
\end{aligned}
$$

where $\tilde{\mathbf{X}}_{N M_{3} P_{2} P_{1} \times P_{0}}^{(3)}$ is a noisy version of $\mathbf{X}_{N M_{3} P_{2} P_{1} \times P_{0}}^{(3)}$. 
TABLE I

CLOSED-FORM SEMI-BLIND KRF RECEIVER FOR $K$ RELAYS

1) A priori information: Measurements tensor $\tilde{\mathcal{X}}^{(K+1)}$, and code matrices $\mathbf{G}_{k}, k=0, \cdots, K$.

2) Symbol estimation: $\mathbf{S}$.

(2.1) Compute the LS estimate $\hat{\mathbf{R}}=\tilde{\mathbf{X}}_{N M_{K+1} P_{K} \cdots P_{1} \times P_{0}}^{(K+1)} \mathbf{G}_{0}^{*}$.

(2.2) Apply the KRF algorithm to estimate the matrix factors $\mathbf{S}$ and $\mathbf{H}_{M_{K+1} P_{K} \cdots P_{1} \times M_{0}}^{(0 \mapsto K+1)}$ from $\hat{\mathbf{R}}$.

(2.3) Remove the scaling ambiguities from $\hat{\mathbf{S}}$ and $\hat{\mathbf{H}}_{M_{K+1} P_{K} \cdots P_{1} \times M_{0}}^{(0 \mapsto K+1)}$ using (23) and (24), respectively.

(2.4) Project the estimated symbols onto the symbol alphabet.

3) Channels estimation: $\mathbf{H}_{k+1}, k=0, \cdots, K$.

Given $\hat{\mathbf{H}}_{M_{K+1} P_{K} \cdots P_{1} \times M_{0}}^{(0 \mapsto K+1)}$ estimated at step 1.2.

For $k=0$ up to $K-1$, repeat

(3.1) Reshape the estimate $\hat{\mathbf{H}}_{M_{K+1} P_{K} \cdots P_{k+1} \times M_{k}}^{(k \mapsto K+1)}$ as $\hat{\mathbf{H}}_{M_{K+1} P_{K} \cdots P_{k+2} M_{k} \times P_{k+1}}^{(k \mapsto K+1)}$, written as in (22), and compute the LS estimate $\hat{\mathbf{Q}}=\hat{\mathbf{H}}_{M_{K+1} P_{K} \cdots P_{k+2} M_{k} \times P_{k+1}}^{\left(k \stackrel{G^{\prime}}{*} \mathbf{G}_{k+1}\right.}$.

(3.2) Apply the KRF algorithm to estimate ${ }^{k+1} \mathbf{H}_{k+1}$ and $\mathbf{H}_{M_{K+1} P_{K} \cdots P_{k+2} \times M_{k+1}}^{(k+1 \mapsto K+1)}$ from $\hat{\mathbf{Q}}$.

(3.3) Remove the scaling ambiguities from $\hat{\mathbf{H}}_{k+1}$ and $\hat{\mathbf{H}}_{M_{K+1} P_{K} \cdots P_{k+2} \times M_{k+1}}^{(k+1 \mapsto K+1)}$ using (25) and (26), respectively.

From the LS estimate $\hat{\mathbf{R}}$ given by Eq. (17), the factors $\mathbf{S}$ and $\mathbf{H}_{M_{3} P_{2} P_{1} \times M_{0}}^{(0 \mapsto 3)}$ are found by the Khatri-Rao product factorization (KRF) algorithm proposed in [16]. Then, the estimate $\hat{\mathbf{H}}_{M_{3} P_{2} P_{1} \times M_{0}}^{(0 \mapsto 3)}$ is reshaped as $\hat{\mathbf{H}}_{M_{3} P_{2} M_{0} \times P_{1}}^{(0 \mapsto 3)}$ to compute $\hat{\mathbf{Q}}$ by means of Eq. (18), from which the factors $\mathbf{H}_{1}$ and $\mathbf{H}_{M_{3} P_{2} \times M_{1}}^{(1 \mapsto 3)}$ are extracted by applying the KRF algorithm. Finally, $\hat{\mathbf{H}}_{M_{3} P_{2} \times M_{1}}^{(1 \mapsto 3)}$ is reshaped as $\hat{\mathbf{H}}_{M_{3} M_{1} \times P_{2}}^{(1 \mapsto 3)}$ to compute $\hat{\mathbf{Z}}$ by means of Eq. (19), from which the channels $\left(\mathbf{H}_{2}, \mathbf{H}_{3}\right)$ are extracted using again the KRF algorithm.

\section{B. General Case}

Let us now consider the general case of $K \geq 2$ relays. The signals received at the destination are given by

$$
\begin{aligned}
\tilde{\mathbf{X}}_{M_{K+1} \times P_{K} P_{K-1} \cdots P_{0} N}^{(K+1)}= & \mathbf{H}_{K+1}\left(\mathbf{G}_{K} \diamond \tilde{\mathbf{X}}_{P_{K-1} \cdots P_{0} N \times M_{K}}^{(K)}\right)^{T} \\
& +\mathbf{N}_{M_{K+1} \times P_{K} P_{K-1} \cdots P_{0} N}^{(K+1)},
\end{aligned}
$$

which defines a $(K+3)$ th-order tensor $\tilde{\mathcal{X}}^{(K+1)} \in$ $\mathbb{C}^{M_{K+1} \times P_{K} \times P_{K-1} \times \cdots \times P_{0} \times N}$

Disregarding the noise terms, let us replace recursively $\mathbf{X}_{P_{K-1} \cdots P_{0} N \times M_{K}}^{(K)}$ as a function of $\mathbf{X}_{P_{K-2} \cdots P_{0} N \times M_{K-1}}^{(K-1)}$, then as a function of $\mathbf{X}_{P_{K-3} \cdots P_{0} N \times M_{K-2}}^{(K-2)}$, and so on, up to $\mathbf{S}$. We obtain the following equivalent third-order PARAFAC model $\left\|\mathbf{H}_{M_{K+1} P_{K} \cdots P_{1} \times M_{0}}^{(0 \mapsto K+1)}, \mathbf{G}_{0}, \mathbf{S} ; M_{0}\right\|$ for $\mathcal{X}^{(K+1)}$

$$
\mathbf{X}_{N M_{K+1} P_{K} \cdots P_{1} \times P_{0}}^{(K+1)}=\left(\mathbf{S} \diamond \mathbf{H}_{M_{K+1} P_{K} \cdots P_{1} \times M_{0}}^{(0 \mapsto K+1)}\right) \mathbf{G}_{0}^{T},
$$

where $\mathbf{H}_{M_{K+1} P_{K} \cdots P_{1} \times M_{0}}^{(0 \mapsto K+1)}$ is a matrix unfolding of the $(K+2)$-th order effective channel tensor $\mathcal{H}^{(0 \mapsto K+1)} \in$ $\mathbb{C}^{M_{K+1} \times P_{K} \times \cdots \times P_{1} \times M_{0}}$ linking the source and the destination, which satisfies the following recurrence relation, for $k=$
$0,1, \cdots, K-1$

$$
\begin{array}{r}
\mathbf{H}_{M_{K+1} P_{K} \cdots P_{k+2} M_{k} \times P_{k+1}}^{(k \mapsto K+1)}=\left(\mathbf{H}_{M_{K+1} P_{K} \cdots P_{k+2} \times M_{k+1}}^{(k+1 \mapsto K+1)}\right. \\
\left.\diamond \mathbf{H}_{k+1}^{T}\right) \mathbf{G}_{k+1}^{T},
\end{array}
$$

This is a third-order PARAFAC model $\left\|\mathbf{H}_{M_{K+1} P_{K} \cdots P_{k+2} \times M_{k+1}}^{(k+1 \mapsto K}, \mathbf{G}_{k+1}, \mathbf{H}_{k+1}^{T} ; M_{k+1}\right\|$ for the contracted form of the effective channel tensor $\mathcal{H}^{(k \mapsto K+1)} \in \mathbb{C}^{M_{K+1} \times P_{K} \times \cdots \times P_{k+1} \times M_{k}}$ linking the node $k$ and the destination, obtained by combining its modes $\left(m_{K+1}, p_{K}, \cdots, p_{k+2}\right)$.

The symbol matrix and the channels $\mathbf{H}_{k}, k=1, \cdots, K+1$, can be estimated in $K+1$ steps. After the computation of the LS estimate $\hat{\mathbf{R}}=\tilde{\mathbf{X}}_{N M_{K+1} P_{K} \cdots P_{1} \times P_{0}}^{(K+1)} \mathbf{G}_{0}^{*}$ deduced from Eq. (21), the symbol matrix $\mathbf{S}$ and the total effective channel $\mathbf{H}_{M_{K+1} P_{K} \cdots P_{1} \times M_{0}}^{(0 \mapsto K+1)}$ are determined by applying the $\mathrm{KRF}$ algorithm to $\hat{\mathbf{R}}$. Then, the channels are estimated in a sequential way by exploiting the recurrence equation (22), initialized with the total effective channel estimate obtained in the first step, and reshaped as $\hat{\mathbf{H}}_{M_{K+1} P_{K} \cdots P_{2} M_{0} \times P_{1}}^{(0 \mapsto K+1)}$. The resulting closed-form semi-blind receiver for $K$ relays is summarized in Table I. Note that $\mathbf{H}^{(K \mapsto K+1)}=\mathbf{H}_{K+1}$. Moreover, in the case $K=2$, the algorithm described in Table I is equivalent to computing the LS estimates (17)-(19), and then applying the KRF algorithm for extracting the matrix factors from the estimated Khatri-Rao products.

\section{IDENTIFIABILITY AND SCALING AMBIGUITY}

In the case $K=2$, the system parameter identifiability is linked to the uniqueness of the least square (LS) estimates of the Khatri-Rao products $\mathbf{R}, \mathbf{Q}$ and $\mathbf{Z}$, i.e. the right-invertibility of $\mathbf{G}_{k}^{T}$, for $k=0,1,2$, in (14)-(16). In the general case ( $K \geq$ 2 ), the uniqueness conditions consist in the right-invertibility of $\mathbf{G}_{0}^{T}$ in (21) and of $\mathbf{G}_{k+1}^{T}$, for $k=0, \cdots, K-1$, in (22), i.e. the full column rank property for $\mathbf{G}_{k}$, for $k=0, \cdots, K$. This implies the necessary conditions $P_{k} \geq M_{k}$, for $k=0, \cdots, K$.

The unknown matrices $\left(\mathbf{S}, \mathbf{H}_{k}, k=1, \cdots, K+1\right)$ estimated from $K+1$ Khatri-Rao products are affected by scaling ambiguities, which can be eliminated by assuming the knowledge of the first row of one factor of each Khatri-Rao product. Herein, we assume the first row of $\mathbf{S}$ and the first column of $\mathbf{H}_{k}$, for $k=1, \cdots, K+1$, are known and equal to the all-one vector. In practice, this a priori knowledge can be determined using short training sequences generated at the relays, as pointed out in [13]. In the case $K \geq 2$, we have the following ambiguity relations

$$
\begin{aligned}
\hat{\mathbf{S}} & \leftarrow \hat{\mathbf{S}} \boldsymbol{\Lambda}_{\mathbf{S}} \\
\hat{\mathbf{H}}_{M_{K+1} P_{K} \cdots P_{1} \times M_{0}}^{(0 \mapsto K+1)} & \leftarrow \hat{\mathbf{H}}_{M_{K+1} P_{K} \cdots P_{1} \times M_{0}}^{(0 \mapsto K+1)}\left(\boldsymbol{\Lambda}_{\mathbf{S}}\right)^{-1} \\
\hat{\mathbf{H}}_{k+1}^{T} & \leftarrow \hat{\mathbf{H}}_{k+1}^{T} \boldsymbol{\Lambda}_{\mathbf{H}_{k+1}^{T}}, \\
\hat{\mathbf{H}}_{M_{K+1} P_{K} \cdots P_{k+2} \times M_{k+1}}^{(k+1 \mapsto K+1)} & \leftarrow \hat{\mathbf{H}}_{M_{K+1} P_{K} \cdots P_{k+2} \times M_{k+1}}^{(k+1 \mapsto K+1)}\left(\boldsymbol{\Lambda}_{\mathbf{H}_{k+1}^{T}}\right)^{-1},
\end{aligned}
$$

where $\boldsymbol{\Lambda}_{\mathbf{S}}=\operatorname{diag}^{-1}\left(\hat{\mathbf{S}}_{1 .}\right)$ and $\boldsymbol{\Lambda}_{\mathbf{H}_{k+1}^{T}}=\operatorname{diag}^{-1}\left(\left(\hat{\mathbf{H}}_{k+1}\right)_{.1}\right)$.

\section{Simulation Results}

In this section, simulation results are provided to evaluate the performance of the proposed semi-blind receiver. The 


$$
\begin{aligned}
\left(\hat{\mathbf{H}}_{M_{K+1} P_{K} \cdots P_{1} \times M_{0}}^{(0 \mapsto K+1)}\right)^{T} & =\left(\left(\mathbf{G}_{0}^{H} \mathbf{G}_{0}\right) *\left(\mathbf{S}^{H} \mathbf{S}\right)\right)^{-1}\left(\mathbf{G}_{0} \diamond \mathbf{S}\right)^{H} \tilde{\mathbf{X}}_{P_{0} N \times M_{K+1} P_{K} \cdots P_{1}}^{(K+1)} \\
& =\operatorname{diag}^{-1}\left(\rho_{1}, \cdots, \rho_{M_{0}}\right)\left(\mathbf{G}_{0} \diamond \mathbf{S}\right)^{H} \tilde{\mathbf{X}}_{P_{0} N \times M_{K+1} P_{K} \cdots P_{1}}^{(K+1)}
\end{aligned}
$$

relays are assumed to be uniformly distributed between the source and the destination. The considered performance criteria are the symbol error rate (SER) and the normalized mean square error (NMSE) of the estimated channels. Each SER curve represents an average over $4 \times 10^{4}$ Monte Carlo runs. Each run corresponds to a realization of all channel and symbol matrices, and noise tensors. The transmitted symbols are randomly drawn from a unit energy quadrature amplitude modulation (QAM) symbol alphabet. The SER curves are plotted as a function of the symbol energy to noise spectral density ratio $\left(E_{s} / N_{0}\right)$. At each run, the variances of the additive noise terms at the relays and the destination are equal. We assume $\mathbf{H}_{k+1}, k=0, \cdots, K$, have i.i.d. zero-mean circularly-symmetric complex Gaussian entries with variances given by $1 / \eta^{\beta} M_{k}$, where $\eta=d / d_{0}=1 /(K+1)$ and $\beta=3$, $d$ denoting the distance between two consecutive nodes, and $d_{0}$ the distance between the source and the destination.

Figure 2 compares the SER vs. $E_{s} / N_{0}$ for three different numbers of relays and two system parameter settings, assuming 4-QAM modulation. The results indicate that additional diversity gains are obtained as the number of relays increases from $K=1$ to $K=3$. These gains come from the additional space-time spreading introduced by the KRST coding at each relay, which results in lower error rates. Moreover, increasing the values of $N, P_{k}$ and $M_{k}$ from 2 to 4 also leads to better SER performance, as expected.

Figure 3 depicts the NMSE associated with the estimation of the individual channels, assuming $K=2$ relays, 4-QAM modulation, and $P_{k}=M_{k}=N=3$. As a reference for comparison, we also show the performance of a supervised LS channel estimation scheme, where $\mathbf{S}$ is a training sequence matrix, with $\rho_{r}=\left\|(\hat{\mathbf{S}})_{. r}\right\|^{2}, r=1, \cdots, M_{0}$. This supervised receiver is based on Eq. (27) with $K=2$, which provides an estimate of the effective channel $\hat{\mathbf{H}}_{M_{3} P_{2} P_{1} \times M_{0}}^{(0 \mapsto 3)}$, without matrix inversion due to the column orthonormality property of $\mathbf{G}_{0}$. The estimates of $\mathbf{H}_{M_{3} P_{2} \times M_{1}}^{(1 \mapsto 3)}$ and $\mathbf{H}_{1}$ are then calculated from $\hat{\mathbf{Q}}$ defined in (18), by applying the KRF algorithm. Finally, the individual channels $\mathbf{H}_{2}$ and $\mathbf{H}_{1}$ are determined from $\hat{\mathbf{Z}}$ defined in (19) by applying once more the KRF algorithm. From Fig. 3, we can conclude that the NMSEs obtained with the proposed semi-blind receiver are very close to the ones provided by the supervised receiver, while being more spectrally efficient. V. CONCLUSION

We have shown that the signals received at the destination node of a one-way multi-hop amplify-and-forward relaying system employing KRST codes at $K$ relays define a $(K+$ 3 )-th order tensor satisfying a generalized nested PARAFAC model. A sequential closed-form semi-blind receiver for jointly estimating the information symbols and the individual channels has been derived by decomposing this model into $K+1$ third-order PARAFAC models. Each step of

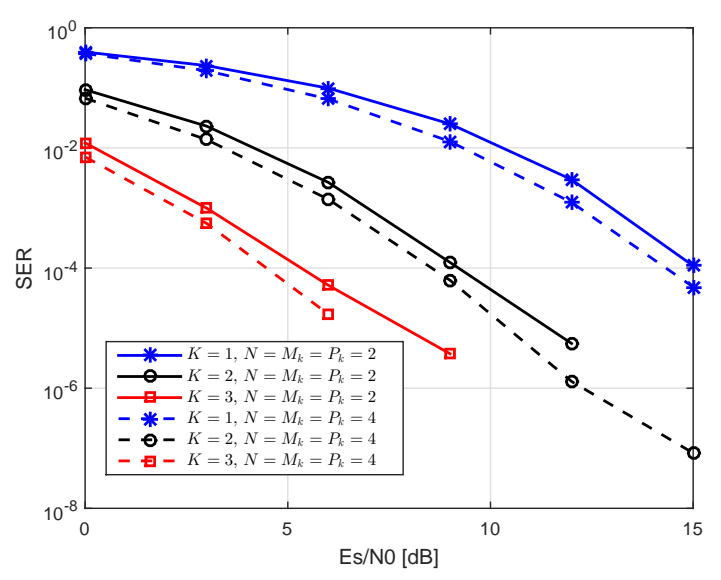

Fig. 2. SER for three different numbers of relays and two system configurations.

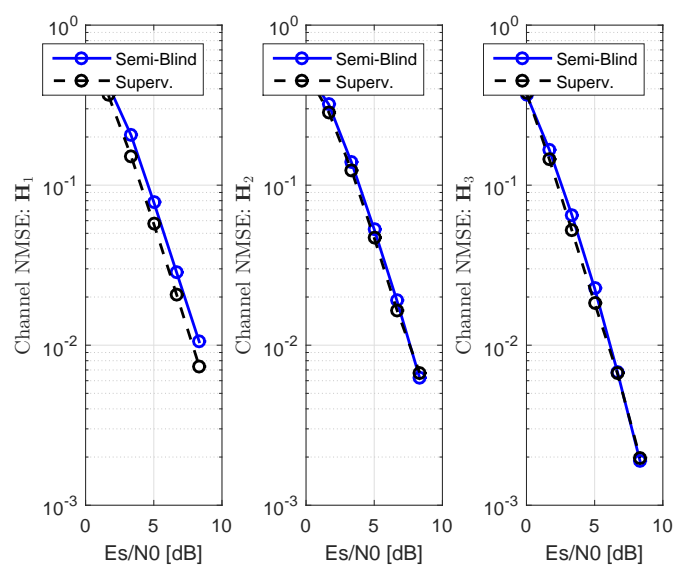

Fig. 3. NMSE of the individual channels with $K=2$.

the proposed algorithm solves a Khatri-Rao factorization problem. Therefore, the dominant complexity of the proposed semi-blind receiver is associated with one singular value decomposition (SVD)-based rank-one approximation for symbols estimation, and $K$ sequential SVD-based rank-one approximations for individual channels estimation.

The choice of truncated DFT coding matrices ensures system parameter identifiability. Simulation results have demonstrated the effectiveness of the proposed multi-hop relay system. Some extensions of this work include the exploitation of the noise structure to develop a tensor-based receiver using a minimum-mean-square-error (MMSE) algorithm to improve the estimation performance, and an extension to the case of orthogonal frequency division multiplexing (OFDM) relay systems. 


\section{REFERENCES}

[1] C. Dehos, J. L. González, A. D. Domenico, D. Kténas, and L. Dussopt, "Millimeter-wave access and backhauling: the solution to the exponential data traffic increase in $5 \mathrm{G}$ mobile communications systems?" IEEE Communications Magazine, vol. 52, no. 9, pp. 88-95, September 2014.

[2] C. X. Wang, F. Haider, X. Gao, X. H. You, Y. Yang, D. Yuan, H. M. Aggoune, H. Haas, S. Fletcher, and E. Hepsaydir, "Cellular architecture and key technologies for 5G wireless communication networks," IEEE Communications Magazine, vol. 52, no. 2, pp. 122-130, February 2014.

[3] J. Du, M. Xiao, and M. Skoglund, "Cooperative network coding strategies for wireless relay networks with backhaul," IEEE Transactions on Communications, vol. 59, no. 9, pp. 2502-2514, September 2011.

[4] T. A. Khan, P. Orlik, K. J. Kim, and R. W. Heath, "Performance analysis of cooperative wireless networks with unreliable backhaul links," IEEE Communications Letters, vol. 19, no. 8, pp. 1386-1389, Aug 2015.

[5] P. Lioliou and M. Viberg, "Least-squares based channel estimation for MIMO relays," in 2008 International ITG Workshop on Smart Antennas, Feb 2008, pp. 90-95.

[6] P. Lioliou, M. Viberg and M. Coldrey, "Efficient Channel Estimation Techniques for Amplify and Forward Relaying Systems," IEEE Transactions on Communications, vol. 60, no. 11, pp. 3150-3155, November 2012.

[7] Y. Rong, M. R. A. Khandaker, and Y. Xiang, "Channel estimation of dual-hop MIMO relay system via parallel factor analysis," IEEE Transactions on Wireless Communications, vol. 11, no. 6, pp. 2224-2233, June 2012.

[8] F. Roemer and M. Haardt, "Tensor-based channel estimation and iterative refinements for two-way relaying with multiple antennas and spatial reuse," IEEE Transactions on Signal Processing, vol. 58, no. 11 , pp. 5720-5735, Nov 2010.

[9] X. Han, A. L. F. de Almeida, and Z. Yang, "Channel estimation for MIMO multi-relay systems using a tensor approach," EURASIP Journal on Advances in Signal Processing, vol. 2014, no. 1, p. 163, 2014.

[10] I. V. Cavalcante, A. L. F. de Almeida, and M. Haardt, "Joint channel estimation for three-hop MIMO relaying systems," IEEE Signal Processing Letters, vol. 22, no. 12, pp. 2430-2434, Dec 2015.

[11] L. R. Ximenes, G. Favier, A. L. F. de Almeida, and Y. C. B. Silva, "PARAFAC-PARATUCK semi-blind receivers for two-hop cooperative MIMO relay systems," IEEE Transactions on Signal Processing, vol. 62, no. 14, pp. 3604-3615, July 2014.

[12] L. R. Ximenes, G. Favier, and A. L. F. de Almeida, "Semi-blind receivers for non-regenerative cooperative MIMO communications based on nested PARAFAC modeling," IEEE Transactions on Signal Processing, vol. 63, no. 18, pp. 4985-4998, Sept 2015.

[13] _ _ "Closed-form semi-blind receiver for MIMO relay systems using double Khatri-Rao space-time coding," IEEE Signal Processing Letters, vol. 23, no. 3, pp. 316-320, March 2016.

[14] N. D. Sidiropoulos and R. S. Budampati, "Khatri-Rao space-time codes," IEEE Transactions on Signal Processing, vol. 50, no. 10, pp. 2396-2407, Oct 2002.

[15] A. L. F. de Almeida and G. Favier, "Double Khatri-Rao space-time-frequency coding using semi-blind PARAFAC based receiver," IEEE Signal Processing Letters, vol. 20, no. 5, pp. 471-474, May 2013.

[16] A. Y. Kibangou and G. Favier, "Non-iterative solution for PARAFAC with a Toeplitz matrix factor," in The 17th European Signal Processing Conference, 2009, pp. 691-695. 